\title{
Intrapulmonary myelolipoma and its computed tomography features: A case report and literature review
}

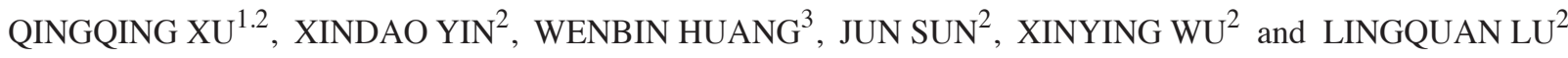 \\ ${ }^{1}$ Department of Radiology, Huai'an First People's Hospital, Nanjing Medical University, Huai'an, Jiangsu 223300; \\ Departments of ${ }^{2}$ Radiology and ${ }^{3}$ Pathology, Nanjing Medical University Affiliated Nanjing Hospital, \\ Nanjing, Jiangsu 210006, P.R. China
}

Received April 1, 2014; Accepted September 26, 2014

DOI: $10.3892 / \mathrm{ol} .2015 .2913$

\begin{abstract}
Intrapulmonary myelolipoma is a rare, benign tumor composed of mature adipose tissue and normal hematopoietic cells. To the best of our knowledge, 10 cases of intrapulmonary myelolipoma, including the present case, have been reported to date, and the majority have focused on the pathological diagnosis of the disease. The radiological features of intrapulmonary myelolipoma have not been studied. Therefore, the present study reports a case of primary myelolipoma in the lung, and examines its computed tomography features and pathology. Furthermore, other potential diagnoses are discussed in the context of the relevant literature. The present report describes the case of a 57-year-old female who experienced chills, but no coughing or expectoration, with an intermittent fever of $38.6^{\circ} \mathrm{C}$ that had been apparent for 13 days. Chest CT scan revealed a benign nodule and bronchiectasis in the lower lobe of the right lung. The patient then underwent a lobectomy of the lower right lung by thoracoscopy. The histological analysis of the excised specimen identifid a myelolipoma consisting of mature adipose tissue and hematopoietic cells. There was no recurrence after 513 days of follow-up, as shown by CT.
\end{abstract}

\section{Introduction}

Myelolipoma is a rare and benign tumor that is typically asymptomatic. The tumor most frequently develops in the adrenal gland, and consists of mature adipose tissue and normal hematopoietic cells (1). Extra-adrenal myelolipomas are extremely rare, particularly in the presacral or perineal space (2). Intrapulmonary myelolipoma is relatively rare; up to now, only 10 cases of intrapulmonary lesions have been

Correspondence to: Dr Xindao Yin, Department of Radiology, Nanjing Medical University Affiliated Nanjing Hospital, 68 Changle Road, Nanjing, Jiangsu 210006, P.R. China

E-mail: yinxindao@sina.com; y.163yy@163.com

Key words: myelolipoma, lung, computed tomography, pathology reported worldwide (3-11). Conservative resection strategies may be appropriate, as recurrence and malignancy have not been reported. In the present study, a case of intrapulmonary myelolipoma is described, and the computed tomography (CT) findings and potential confounding characteristics that may lead to incorrect diagnoses are reviewed. Informed consent was obtained from the patient.

\section{Case report}

Patient presentation. A 57-year-old female was admitted to the Nanjing Medical University Affiliated Nanjing Hospital (Nanjing, Jiangsu, China), with an intermittent fever of $38.6^{\circ} \mathrm{C}$ that had been apparent for 13 days. The patient experienced chills, but no coughing or expectoration. A chest CT scan performed at a local hospital revealed the presence of a nodule, bronchiectasis and an infection in the lower lobe of the right lung. Subsequent to treatment with $3,200,000$ IU of patulin and antibiotics every $8 \mathrm{~h}$ for 5 days (orally), the patient underwent a follow-up chest CT scan at the Nanjing Medical University Affiliated Nanjing Hospital, which revealed a benign nodule and bronchiectasis in the lower lobe of the right lung. The tumor was observed in the tributary of the lower right pulmonary vein, but had not altered in size during the time between the two CT scans. The results of routine blood work appeared within normal limits. The patient then underwent a lobectomy of the lower right lung by thoracoscopy. The histological analysis of the excised specimen identified a myelolipoma consisting of mature adipose tissue and hematopoietic cells.

CT procedures. A single-spiral CT scanner (Asteion; Toshiba Medical Systems, Tokyo, Japan) was used to perform the chest scan, using the following parameters: Tube voltage, $120 \mathrm{kV}$; tube current, $140 \mathrm{MA}$; window width, 1,700 Hounsfield units (HU); window level, -550 HU; and matrix size, 480x480 pixels. The CT slice thickness and interval were each $10 \mathrm{~mm}$. The CT image was obtained from the apex to the base of the lung, with the patient at full end-inspiration and in the supine position.

$C T$ findings. The axial CT images of the chest revealed a smooth and well-defined nodule of heterogeneous 
Table I. Patient and tumor characteristics of reported intrapulmonary myelolipomas.

\begin{tabular}{|c|c|c|c|c|c|c|c|}
\hline Case & First author/s (ref.) & $\begin{array}{l}\text { Age, years/ } \\
\text { gender }\end{array}$ & $\begin{array}{l}\text { Patient } \\
\text { history }\end{array}$ & $\begin{array}{l}\text { Tumor } \\
\text { location }\end{array}$ & $\begin{array}{c}\text { Number of } \\
\text { sites }\end{array}$ & Size, $\mathrm{cm}$ & $\begin{array}{c}\text { Diagnosis } \\
\text { method }\end{array}$ \\
\hline 1 & Saleeby (11) & $81 / \mathrm{F}$ & Pneumonia & Peripherally & Single & $\phi 2.5$ & Autopsy \\
\hline 2 & Hunter et al (4) & $70 / \mathrm{F}$ & RA, steroid & Peripherally & Multiple & ND & Biopsy \\
\hline 3 & Ziolkowski et al (9) & $\begin{array}{l}\text { 49/M, } \\
59 / \mathrm{M}\end{array}$ & Pneumonia & $\begin{array}{l}\text { LLL, } \\
\text { RLL }\end{array}$ & Multiple & $\begin{array}{c}7 \times 5 \times 5 \\
\phi 2\end{array}$ & $\begin{array}{l}\text { Autopsy, } \\
\text { resection }\end{array}$ \\
\hline 4 & Zunarelli et al (10) & $52 / \mathrm{M}$ & MGUS, BC & RLL & Single & ND & Lobectomy \\
\hline 5 & Sabate and Shahian (7) & $54 / \mathrm{M}$ & $\mathrm{HC}$ & LUL & Single & $\phi 2.5$ & Enucleation \\
\hline 6 & Sato et al (8) & $71 / \mathrm{M}$ & Lung cancer & LLL & Single & $\phi 2.0$ & Autopsy \\
\hline 7 & Lu and Xiao (6) & $45 / \mathrm{M}$ & Pneumonia & LUL & Single & $\phi 1.5$ & Lobectomy \\
\hline 8 & Lin et al (5) & $45 / \mathrm{M}$ & ND & LUL & Single & $4.5 \times 3.5 \times 2.3$ & ND \\
\hline 9 & Huang et al (3) & $53 / \mathrm{M}$ & Pneumonia, atelectasis & LL & Single & $2.3 \times 1.2 \times 1.0$ & Biopsy \\
\hline 10 & Present case & $57 / \mathrm{F}$ & Bronchiectasis & RLL & Single & $\phi 1.6$ & Lobectomy \\
\hline
\end{tabular}

F, female; M, male; P, peripherally; RA, rheumatoid arthritis; MGUS, monoclonal gammopathy of undetermined significance; BC, bronchial carcinoid tumor; HC, hypercholesterolemia; LLL, left lower lobe; LUL, left upper lobe; ND, not determined; RLL, right lower lobe; RUL, right upper lobe; LL, left lung.
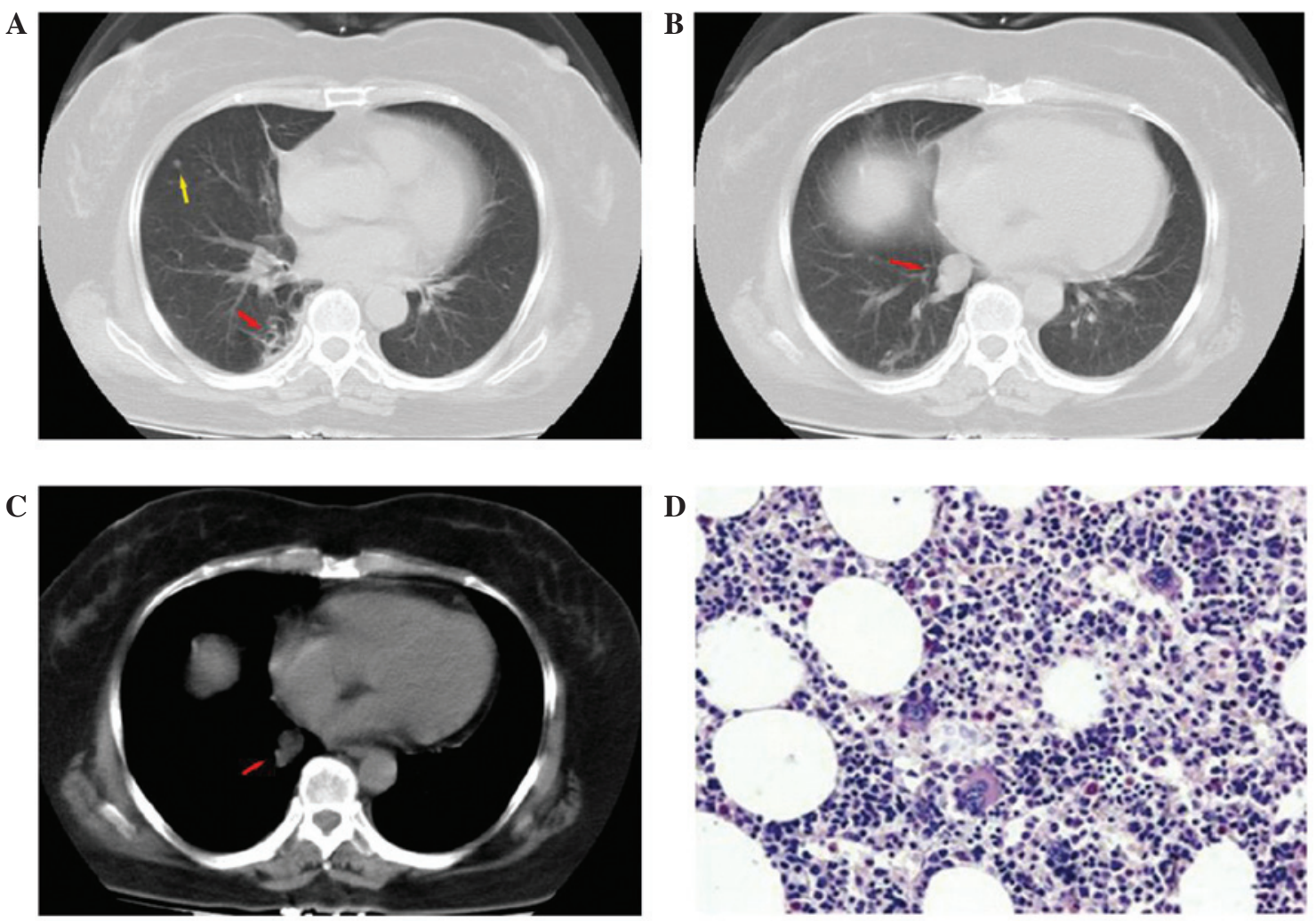

Figure 1. (A) Chest computed tomography (CT) (lung window). CT revealing bronchiectasis (red arrow) and small nodules (yellow arrow), with infection in the lower lobe of the right lung. (B) Chest CT (lung window). CT revealing a small nodule (red arrow) in the lower right lung. (C) Chest CT (mediastinal window). CT revealing a small nodule (red arrow) in the right lower lung, with the density of fat. (D) Hematoxylin and eosin staining revealing that the tumor was composed of mature adipose tissue and hematopoietic cells.

composition in the medial segment of the lower lobe of the right lung. The largest area of the nodule in the axial plane was $\sim 14 \times 15 \mathrm{~mm}$. Negative density values were observed in the mass, with an average density of $-46 \mathrm{HU}$ (range, -30 to $-68 \mathrm{HU}$ ). The mass was adjacent to the tributary of the lower right pulmonary vein. No density shadow, indicating calcification, was detected in the nodule by CT (Fig. 1A and B), and cystic bronchiectasis was present within the adjacent lung (Fig. 1C).

Pathological findings. Grossly, the nodule was grayish-red and measured $16 \mathrm{~mm}$ in diameter at its largest point. Light microscopy revealed that the tumor was composed of mature adipose 
tissue and normal mature hematopoietic cells, including myeloid, erythroid and megakaryocytic cells, in normal proportions. No trabecular bone, calcification or ossification was observed in the tumor (Fig. 1D).

\section{Discussion}

Myelolipoma was first described in the early 20th century, and is usually observed in the adrenal gland (1). The occurrence of extra-adrenal myelolipomas is less common, however, they have been observed in the presacral soft tissue, retroperitoneum, spleen, liver, stomach, mediastinum and nasal cavity $(2,12-15)$. Intrapulmonary myelolipoma is rare, and to the best of our knowledge, only 10 cases (including three cases reported in the Chinese literature and the present case) have been documented (3-11). The patients in these cases ranged in age between 45 and 81 years, and were predominately male. Table 1 summarizes the patient and tumor characteristics of the reported intrapulmonary myelolipomas, including the data from the present study. A diagnosis of intrapulmonary myelolipoma is usually made when abnormal ratios of mature adipose tissue and hematopoietic cells, including myeloid, erythroid and megakaryocytic elements, and occasionally lymphocytes, are observed histologically $(2,4,10,11,15)$.

In total, eight of the 10 previously reported cases of intrapulmonary myelolipoma were solitary, and two were multifocal. Intrapulmonary myelolipomas are usually small nodules measuring $<30 \mathrm{~mm}$ at their largest dimension, however, one case revealed a nodule of $7 \mathrm{~cm}$ in length (15). Patients with intrapulmonary myelolipoma usually present with a history of pneumonia, however, one literature case presented with a bronchial carcinoid tumor (10), and the patient in the present study presented with a history of pneumonia and bronchiectasis. The CT features observed in the present case study were as expected, with the lesions typically possessing negative HU values from macroscopic fat. Due to the intermixed hematopoietic tissue, the attenuation of macroscopic fat is usually heterogeneous and higher than that of retroperitoneal fat, as was demonstrated in this patient. Regions of higher density, due to hemorrhage and calcification, would also be observed. The tumors may also be mildly or moderately enhanced following administration of intravenous contrast agents. Upon magnetic resonance imaging, lesions demonstrate high signal intensity from mature adipose on T1- and T2-weighted imaging. Fat suppression imaging also reduces signal intensity in the fatty components of lesions (16). In addition, myeloid elements demonstrate low signal intensity upon T1-weighted imaging, and intermediate intensity upon T2-weighted imaging.

$\mathrm{CT}$ reveals certain intrapulmonary myelolipomas to be centrally located masses, leading to obstructive pneumonia and atelectasis in the lobe or lung segment, and a frequent misdiagnosis of lung cancer. In these cases, accurate diagnoses are difficult, even following histological examination of the biopsy specimens obtained by bronchoscopy $(3,6)$. Upon CT, negative HU values of adipose components should distinguish myelolipomas from lung cancer. Intrapulmonary myelolipomas located in the periphery of the lung, which demonstrate adipose densities upon CT, are usually small masses with smooth, heterogeneous and well-defined borders (6). This was the clinical presentation of the patient in the present study.

Intrapulmonary myelolipomas must be differentiated from phlebangiomas, hamartomas, lipomas and teratomas. Intrapulmonary phlebangiomas are rare tumors, which are often enhanced significantly following administration of intravenous contrast agents (17). In the present case study, without CT to identify the lipid component of the lesion, the nodule in the tributary of the lower-right pulmonary vein could potentially have been be misdiagnosed as a phlebangioma. Intrapulmonary hamartomas are common, benign masses in the pulmonary parenchyma. The histological makeup of these tumors is characterized by a fibromyxoid stroma, cartilage, bronchial cells, adipose tissue and bone. The characteristic 'popcorn-like' calcification that occurs with these masses is frequently observed by CT (18). Intrapulmonary lipomas are exceptionally rare. Only 10 cases have been reported to date in Medline, and no marked gender differences are evident with respect to these tumors $(19,20)$. Pathologically, intrapulmonary lipomas are grossly observed as being well-defined, thinly-encapsulated and rounded, pale-yellow masses, which are composed of mature adipose tissue upon microscopic analysis (19). A homogeneous and defined lesion that contains a high density of lipid can be revealed by CT imaging. No contrast enhancement is observed with these masses, as they lack soft tissues $(19,20)$. Myelolipomas that are predominantly composed of fatty tissue are difficult to distinguish from lipomas. Intrapulmonary teratomas may be malignant and upon histopathology, contain tissue that originates from any of the three germinal layers. Calcification is a typical radiological finding and is extremely valuable for clinical diagnoses when CT reveals discrete areas of soft tissue, fat, fluid or punctate calcification (20). In the case that peripheral myelolipomas in the lung are mainly composed of hematopoietic cells, it may be necessary to perform a percutaneous needle biopsy for a definite diagnosis.

In general, accurate identification of intrapulmonary myelolipoma depends on histopathology. However, it is important to understand the radiological characteristics, which can provide valuable diagnostic clues in clinical practice. Although it is difficult to achieve an accurate diagnosis, a chest CT scan demonstrating a well circumscribed nodule with low attenuation of adipose tissue is helpful in the diagnosis of intrapulmonary myelolipomas. Conservative resection strategies may be appropriate, as recurrence and malignancy have not been reported. However, given the likelihood of subsequent hemorrhage or compression of adjacent bronchi by myelolipomas, surgical removal is the ideal choice.

\section{Acknowledgements}

The authors would like to thank Professor Wenbin Huang, Department of Pathology, Nanjing Medical University Affiliated Nanjing Hospital (Nanjing First Hospital) for providing assistance with the pathological analysis.

\section{References}

1. Osborn M, Smith M, Senbanjo T, et al: Adrenal myelolipoma - clinical, radiological and cytological findings: a case report. Cytopathology 13: 242-246, 2002. 
2. Beiko D, Roldan H, Sengupta SK and George RL: Laparoscopic excision of a large extra-adrenal perirenal myelolipoma. Can Urol Assoc J 4: E39-E41, 2010.

3. Huang WT, Zhao SJ and Lin DM: Pulmonary-bronchus myelolipoma and review on extra-adrenal myelolipomas in Chinese literature. Chin Med J (Engl) 125: 3188-3190, 2012.

4. Hunter SB, Schemankewitz EH, Patterson C and Varma VA: Extraadrenal myelolipoma. A report of two cases. Am J Clin Pathol 97: 402-404, 1992.

5. Lin XY, Song YH and Wang JY: Cystic lung myelolipoma with bone and cartilage metaplasia: A clinicopathological observation. J Diag Pathol 16: 55-57, 2009.

6. Lu X and Xiao L: Myelolipoma of the lung: a case report. Chin Med J (Engl) 116: 951-953, 2003.

7. Sabate CJ and Shahian DM: Pulmonary myelolipoma. Ann Thorac Surg 74: 573-575, 2002.

8. Sato K, Ueda Y, Katsuda S and Tsuchihara K: Myelolipoma of the lung: a case report and brief review. J Clin Pathol 60: 728-730, 2007.

9. Ziókowski P, Muszczyńska-Bernhard B and Dziegiel P: Myelolipoma: the report of two cases in pulmonary location. Pol J Pathol 47: 141-142, 1996.

10. Zunarelli E, Criscuolo M, Malavolta L, Piccinini L and Barbolini G: A case of lung myelolipomatosis in a patient with bronchial carcinoid. Panminerva Med 41: 175-178, 1999.

11. Saleeby ER: Heterotopia of the bone marrow without apparent cause. Am J Pathol 1: 69-76.3, 1925.
12. Arpino L, Capuano $C$ and Franco A: Intradural thoracic myolipoma. ANZ J Surg 81: 843, 2011.

13. George SA, Manipadam MT and Thomas R: Primary myelolipoma presenting as a nasal cavity polyp: a case report and review of the literature. J Med Case Rep 6: 127, 2012.

14. Radhi J: Hepatic myelolipoma. J Gastrointestin Liver Dis 19: 106-107, 2010.

15. Vaziri M, Sadeghipour A, Pazooki A and Shoolami LZ: Primary mediastinal myelolipoma. Ann Thorac Surg 85: 1805-1806, 2008.

16. Guo YK, Yang ZG, Li Y, et al: Uncommon adrenal masses: $\mathrm{CT}$ and MRI features with histopathologic correlation. Eur J Radiol 62: 359-370, 2007.

17. Mao Q, Xiang ST, Bao YM , et al: Pulmonary phlebangiomas under the upper ulmonary vein: A case report. Chin J Radiol 38: 1006, 2004 (In Chinese).

18. Madan K, Sharma S, Singh N and Radhika S: Large pulmonary hamartoma with 'popcorn' like calcification. Monaldi Arch Chest Dis 75: 243-244, 2011.

19. Moran AM, Jian B, Min H, Pechet T and Fogt F: Peripheral intrapulmonary lipoma in a 26-year-old woman - a case report. Pol J Pathol 62: 113-115, 2011.

20. Rana SS, Swami N, Mehta S, Singh J and Biswal S Intrapulmonary teratoma: an exceptional disease. Ann Thorac Surg 83: 1194-1196, 2007. 